\title{
Direct reconstruction of the dark energy scalar-field potential
}

\author{
Chao Li, ${ }^{1}$ Daniel E. Holz, ${ }^{2,3}$ and Asantha Cooray ${ }^{4}$ \\ ${ }^{1}$ California Institute of Technology, Mail Code 130-33, Pasadena, California 91125, USA \\ ${ }^{2}$ Theoretical Division, Los Alamos National Laboratory, Los Alamos, New Mexico 87545, USA, \\ and Department of Astronomy \& Astrophysics, University of Chicago, Chicago, Illinois 60637, USA \\ ${ }^{3}$ The Observatories of the Carnegie Institution of Washington, Pasadena, California 91101, USA \\ ${ }^{4}$ Center for Cosmology, Department of Physics and Astronomy, University of California, Irvine, California 92697, USA
}

(Received 28 November 2006; published 3 May 2007)

\begin{abstract}
While the accelerated expansion of the Universe is by now well established, an underlying scalar-field potential possibly responsible for this acceleration remains unconstrained. We present an attempt to reconstruct this potential using recent $\mathrm{SN}$ data, under the assumption that the acceleration is driven by a single scalar field. Current approaches to such reconstructions are based upon simple parametric descriptions of either the luminosity distance or the dark energy equation of state. We find that these various approximations lead to a range of derived evolutionary histories of the dark energy equation of state (although there is considerable overlap between the different potential shapes allowed by the data). Instead of these indirect reconstruction schemes, we discuss a technique to determine the potential directly from the data by expressing it in terms of a binned scalar field. We apply this technique to a recent SN data set, and compare the results with model-dependent approaches. In a similar fashion to direct estimates of the dark energy equation of state, we advocate direct reconstruction of the scalar-field potential as a way to minimize prior assumptions on the shape, and thus minimize the introduction of bias in the derived potential.
\end{abstract}

DOI: 10.1103/PhysRevD.75.103503

PACS numbers: 98.80.Es, 97.60.Bw, 98.80.Cq

\section{INTRODUCTION}

Distance estimates to type Ia supernovae ( $\mathrm{SNe}$ ) are currently a preferred probe of the expansion history of the Universe [1], and have led to the discovery that the expansion is accelerating [2]. It is now believed that a mysterious dark energy component, with an energy density $\sim 70 \%$ of the total energy density of the Universe, is responsible for the accelerated expansion [3]. While the presence of acceleration is now well established by various cosmological probes, the underlying physics remains a complete mystery. As the precise nature of the dark energy has profound implications, understanding its properties is one of the biggest challenges today.

With the advent of large surveys for type Ia supernovae, such as the Supernova Legacy Survey (SNLS) [4] and Essence [5], among others, it is hoped that we will study details of the expansion, and thereby elucidate the physics responsible for the acceleration. Under the assumption that the dark energy is due to a single scalar field rolling down a potential, several studies have considered how future data might be used to reconstruct the potential, either based on various analytical descriptions of the luminosity distance [6], or through specific assumptions about the potential, such as a polynomial function in the scalar field [7]. It is already well established that certain parametric descriptions of the distance lead to biased estimates for the dark energy equation of state (EOS) and the potential [8,9]. While improved parametric forms of fitting functions have been suggested [10,11], it is unclear how to select an optimal approach for reconstructing the dark energy scalar-field potential from $\mathrm{SN}$ distances (for a review of various possibilities, see Ref. [12]).

In this paper we discuss issues related to potential and dark energy EOS reconstruction by making use of a recent set of SN data from the SNLS survey [13]. The sample includes 73 high-redshift SNe complemented with a sample of 44 nearby supernovae $[13,14]$. We compare and contrast a variety of methods to reconstruct the potential and the dark energy EOS. We write the luminosity distance either as a simple polynomial expansion in redshift, or as a Padé approximation [15] (which avoids some of the known problems in the polynomial expansion when taking derivatives $[8,10,16])$. In addition to approximating the luminosity distance, we also explore two approximations to the EOS: $w(z)=w_{0}+w_{a}(1-a)[17,18]$ and $w(z)=$ $w_{0}-\alpha \ln (1+z)[10]$.

Based on our model reconstruction of the potential, we find that while there is significant overlap of the allowed $V(\phi)$ region favored by each of the four reconstruction methods, the models give rise to different histories for the EOS, especially within the two parameter plane, $w-w^{\prime}$ (the EOS parameter, $w$, and its time derivative, $w^{\prime} \equiv d w / d \ln a$, as functions of redshift [19]). We argue that existing parametric fitting functions for either distance or the EOS lead to biased reconstructions of the potential. In the literature, however, there exist model-independent approaches to the reconstruction of the dark energy density [20] and the EOS [21], which bin the parameters directly as a function of redshift, with the number and width of the bins determined by the statistical quality of data. These estimates can also be arranged to be uncorrelated [21], allowing unique in- 
sights into the evolution without being subject to prior assumed redshift dependencies.

Here we suggest a similar model-independent approach to the reconstruction of the scalar potential from SN data, under the assumption of a single scalar-field potential. Instead of utilizing a polynomial expansion for the potential [7], which assumes a limited range of models (once the expansion is truncated at a certain order), we propose a binning scheme for the potential that can be applied to data with a minimal, and easily controlled and understood, number of assumptions for the potential shape.

The paper is organized as follows: In Sec. II we review techniques for reconstructing the scalar-field potential from SN distances. We also reconstruct the EOS as a function of the redshift, and use this to study the $w-w^{\prime}$ plane (which has been advocated as a way to characterize the underlying potential responsible for the dark energy component by separating the regime into "freezing" and "thawing" potentials [19]; see, also [22] for a Monte Carlo exploration). In Sec. III we explore the impact of different parametrizations on the derived evolutionary histories. While we observe these differences with $\sim 115 \mathrm{SN}$ data points, future large SN data sets may lead to apparently inconsistent results. In Sec. IV, following the approach to model-free estimates of the dark energy EOS [21], we present a model-independent estimate of the scalar-field potential. We conclude with a summary of our main results in Sec. V.

\section{POTENTIAL VIA PARAMETRIC FORMS}

For this study we make use of SN data from SNLS [13]. Because of complications related to independent data sets (e.g., differing calibration, color correction, extinction correction, etc.), we do not attempt to increase the sample size by combining other $\mathrm{SN}$ data sets. The measurements from Ref. [13] present the quantity $\mu_{B}=m_{B}-M$ for $117 \mathrm{SNe}$, with 73 of these at redshifts greater than 0.2 [23] This distance modulus is related to the luminosity distance through $\mu_{B}=5 \log _{10} d_{L}$, while the luminosity distance is related to the comoving radial distance via $d_{L}=c(1+$ $z) r(z) / H_{0}$, where $r(z)=\int_{0}^{z} d z^{\prime} / H\left(z^{\prime}\right)$ with $H(z)$ the expansion rate of the Universe. When model fitting the data, we fix $M=19.3 \pm 0.03$ to the value determined by SNLS. We take the central value; further uncertainty will be incorporated into $\sigma_{\text {int }}$, as discussed below.

In our reconstruction of the potential, we describe $r(z)$ through two parametric forms widely used in the literature. First, we expand $r(z)$ as a simple power law [6] such that

$$
r(z)=z+a_{2} z^{2}+a_{3} z^{3}+a_{4} z^{4} .
$$

Note that the coefficient of the first order term is exactly one. Since this polynomial expansion has known problems when estimating the derivatives of $r(z)$ (e.g., Fig. 3 of Ref. [10], and also Ref. [24]), we also consider a Padé form for $r(z)$ with Ref. [15]:

$$
r(z)=2 \frac{z+c_{1}(1-\sqrt{1+z})}{c_{2}(1+z)+c_{3} \sqrt{1+z}+2-c_{1}-c_{2}-c_{3}},
$$

such that as $z \rightarrow 0, r(z) \rightarrow z$. In this form, using $r(z \rightarrow \infty)$, one can additionally constrain the parameters with:

$$
\begin{aligned}
3 \Omega_{M} & \leq \frac{4 c_{2}+2 c_{3}-c_{1}}{2-c_{1}}, \\
1 & \leq \frac{1}{c_{2}} \leq \frac{1}{2} \int_{1}^{\infty} \frac{d x}{\sqrt{1-\Omega_{M}+\Omega_{M} x^{3}}} .
\end{aligned}
$$

In addition to the two fitting forms for $r(z)$, we also determine $r(z)$ through model parametrizations for $w(z)$, including $w(z)=w_{0}+(1-a) w_{a}[17,18]$ and $w(z)=$ $w_{0}+\alpha \ln (1+z)$ [10]. Since from $w$ it is possible to determine the distance, these approximations allow us to once again reconstruct the dark energy potential.

In each of two parametric descriptions of $r(z)$ we have three free parameters. We parametrize $w(z)$ with two parameters, and include $\Omega_{m}$ as a third free parameter (under the assumption of a flat universe; weakening this assumption significantly degrades our ability to measure anything about the potential with existing data). When showing results related to potentials or EOS as a function of redshift, we take a prior on $\Omega_{m}$ such that the probability is Gaussian with a mean of 0.25 and a standard deviation given by $\sigma=0.05$ [3]. In each case, to obtain the joint likelihood distribution of the parameters given the data, we perform a likelihood analysis:

$$
\chi^{2}\left(p_{i}\right)=\sum_{i=1}^{N} \frac{\left[\mu-\mu_{B}\left(z_{i}\right)\right]^{2}}{\sigma_{\mu_{B}}^{2}+\sigma_{\mathrm{int}}^{2}},
$$

where, following Ref. [13], in addition to statistical uncertainty in $\mu_{B}$ we include an additional Gaussian uncertainty, $\sigma_{\text {int }}=0.13$, representing the intrinsic dispersion of $\mathrm{SN}$ absolute magnitudes, $M$. We ignore complications related to covariances in the Hubble diagram, either due to effects related to calibration [25] or fundamental limitations such as gravitational lensing correlation of SN flux [26] or peculiar velocities [27]. The posterior probability distribution is taken to be $P\left(p_{i} \mid \mu\right) \propto e^{-(1 / 2) \chi^{2}\left(p_{i}\right)}$, and we marginalize the likelihood over the uncertainty in $\Omega_{m}$, assuming a Gaussian prior distribution.

Once the joint probability distribution for parameters is determined, we sample the $1 \sigma$ and $2 \sigma$ range allowed by these parameters to draw a fixed $(>600)$ number of independent $r(z)$ curves consistent with the data. For each of these distance curves, $r_{i}(z)$, we obtain the scalarfield potential, in dimensionless units such that $\tilde{V}(\tilde{\phi})=$ $V(\phi) / \rho_{\text {crit }}=V /\left(3 H_{0}^{2} / 8 \pi G\right)$, through Ref. [6] 


$$
\tilde{V}(\tilde{\phi})=\left[\frac{1}{(d \tilde{r} / d z)^{2}}+\frac{1+z}{3} \frac{d^{2} \tilde{r} / d z^{2}}{(d \tilde{r} / d z)^{3}}\right]-\frac{1}{2} \Omega_{M}(1+z)^{3},
$$

where $\tilde{r}=H_{0} r$. For each of the $r_{i}(z)$ estimates, we also randomly draw $\Omega_{m}$ from a Gaussian prior distribution as described above. The mapping between $z$ and $\phi$, the scalarfield value, is obtained through

$$
\begin{aligned}
\frac{d \tilde{\phi}}{d z}= & -\frac{d \tilde{r} / d z}{(1+z)}\left[-\frac{1}{4 \pi} \frac{(1+z) d^{2} \tilde{r} / d z^{2}}{(d \tilde{r} / d z)^{3}}\right. \\
& \left.-\frac{3}{8 \pi} \Omega_{M}(1+z)^{3}\right]^{1 / 2},
\end{aligned}
$$

where $\tilde{\phi}=\phi / m_{\mathrm{Pl}}$. Furthermore, for models where we parametrize $r(z)$, we can also extract the dark energy EOS as

$$
w(z)=\frac{1+z}{3} \frac{3 \Omega_{m}(1+z)^{2}+2\left(d^{2} r_{i} / d z^{2}\right) /\left(d r_{i} / d z\right)^{3}}{\Omega_{m}(1+z)^{3}-\left(d r_{i} / d z\right)^{-2}}-1 .
$$

When selecting models associated with scalar fields, we require that $d \tilde{\phi} / d z>0$, such that $w \geq-1$. Even in the case of $w(z)$ parametrizations where model fits allow $w<$ -1 , we ignore $w(z)$ below this value as single scalar-field models do not naturally give rise to such EOS.

\section{BIASES IN MODEL-DEPENDENT ESTIMATES}

In Fig. 1 we show the Hubble diagram for the 115 data points from Ref. [13] used in this analysis. For reference, we also plot $\sim 300$ distance curves which are $2 \sigma$ consistent curves drawn from the likelihood distribution for parameters under the Taylor expansion for $r(z)$. The best-fit model with this parametrization has a chi-square value of 113.1 with 112 degrees of freedom. Note that in Ref. [13], $\sigma_{\text {int }}$ is tuned so that $\chi^{2}=1$ for the best-fit model under standard- $\Lambda \mathrm{CDM}$ cosmological fits to the data. We use their best-fit value, $\sigma_{\text {int }}=0.13$, and do not take this intrinsic uncertainty as an additional free parameter in our modeling. The exact value of the intrinsic dispersion does not impact our comparison of different approaches to the reconstruction of the quintessence potential. It is to be emphasized that all of our parametrizations of either distance or the EOS yield comparable $\chi^{2}$ values for the bestfit model. This suggests that all four of the reconstruction methods outlined above are indistinguishable within the redshift range considered.

As discussed in the previous section, for each of the four parametrizations we determine a best-fit $r(z)$ to the $\mathrm{SN}$ data. We then Monte Carlo generate models within $2 \sigma$ of this best-fit, generating over 600 instances of $w(z)$ and $V(\phi)$, all of which are consistent with the underlying SN data set at the $2 \sigma$ level. In Fig. 2 we show the potentials reconstructed from each of the four methods, with the

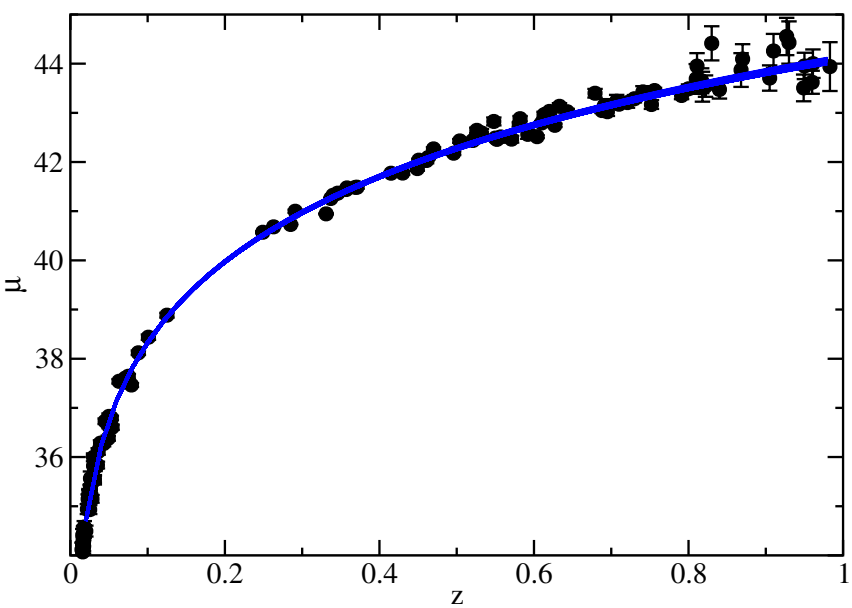

FIG. 1 (color online). Hubble diagram for 115 type Ia SNe used in the present analysis. The error bars are on $\mu_{B}$ only. We also include an additional constant error, $\sigma_{\text {int }}=0.13$, to account for the $\mathrm{SN}$ intrinsic dispersion. For reference we also plot 300 curves drawn uniformly from the $2 \sigma$ consistent likelihood fits to the data using the Taylor expansion with $r(z)=z+a_{2} z^{2}+$ $a_{3} z^{3}+a_{4} z^{4}$.

bands encapsulating $95 \%$ of the distribution of the individual models. Because of the behavior of the Taylor expansion at high $z$, and the fact that we do not restrict the coefficients of the polynomial expansion to follow a flat universe, this parametrization gives rise to a large range of acceptable potentials which satisfy the data. The Padé parametrization of $r(z)$, as well as the $w(z)$ models, significantly improve the constraints on allowed potential shapes. This is because the parameters in the Padé approximation are additionally constrained to satisfy criteria related to the behavior of $r(z)$ as $z \rightarrow \infty$, as well as by the assumption of a flat universe [15]. When fitting the $w(z)$ parametrizations to the data, we were able to impose a prior on $\Omega_{m}$ based on existing cosmological information [this was not possible when using $r(z)$ fitting functions]. While we find some overlap in the $2 \sigma$ allowed region in the $\phi-$ $V(\phi)$ plane between the four approaches, there are also noticeable inconsistencies. Analysis of an identical data set with different approximations to $V$ or $w$ lead to differing resulting best-fit potentials.

The differences related to potential shapes between the four methods are best captured in terms of evolutionary histories for the dark energy EOS. In Fig. 3 we summarize the best-fit $w(z)$ results for each of the four reconstruction techniques. Note that some of our parametrizations allow $w(z)<-1$, but due to our assumption that the dark energy arises from a scalar-field potential where $w(z)$ is always expected to be greater than -1 , we restrict the allowed parameter space to be the region where $w(z)>-1$. Similarly to Fig. 2, we find considerable overlap between different reconstruction schemes in the $w(z)$ versus redshift plane, with most models indicating that as the redshift is decreased, $w(z)$ tends to values between -0.8 and -1.0 at 
CHAO LI, DANIEL E. HOLZ, AND ASANTHA COORAY

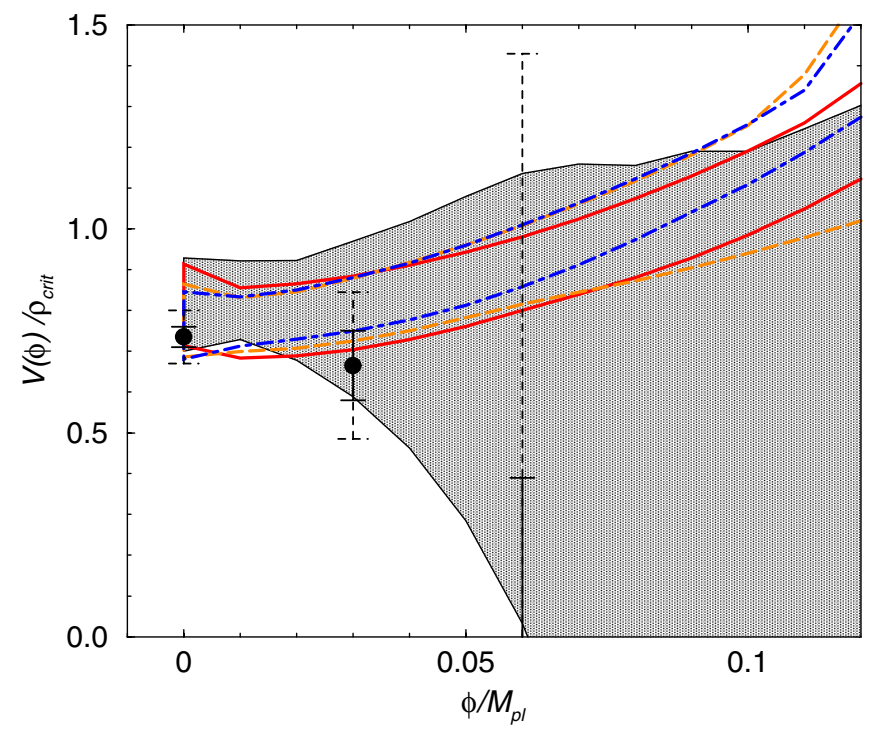

FIG. 2 (color online). The normalized quintessence potential $\tilde{V}(\phi)$ vs $\phi / m_{\mathrm{Pl}}$. The shaded region is allowed at the $2 \sigma$ confidence level when using the Taylor expansion for $r(z)$. The solid lines mark the same when using a Padé approximation to the distance. The dashed and dot-dashed lines are for the cases where $w(z)$ is parametrized by $w(z)=w_{0}+\alpha \ln (1+z)$ and $w(z)=w_{0}+w_{a}(1-a)$, respectively. Here $\phi=0$ corresponds to $z=0$, while $\phi>0.1$ generally corresponds to $z>1$ (depending on $d \phi / d z$ ). The points with error bars show the $1 \sigma$ (solid) and $2 \sigma$ (dashed) model-independent estimates of the potential described in Sec. IV [see Eq. (8)]. While there is considerable overlap in the allowed region, there are also significant differences in terms of the redshift evolution of the EOS.

$z=0$. In terms of our direct $w(z)$ parametrizations, with $w(z)=w_{0}+(1-a) w_{a}$ we find $w_{0}=-1.12 \pm 0.14$ and $w_{a}=0.38 \pm 0.49$ at the $68 \%$ confidence level. In the case of $w(z)=w_{0}+\alpha \ln (1+z)$ we find $w_{0}=-1.08 \pm 0.11$ and $\alpha=0.35 \pm 0.75$. As shown in prior studies [16], $w(z)$ parametrizations allow for a minimum $w(z)$ region at a certain pivot redshift. For the data set used here, this pivot redshift is at $z \sim 0.12$, and at the $2 \sigma$ confidence level we find that the pivot point satisfies $-1.23<w_{p} \equiv w(z=$ $0.12)<-0.74$, using $w(z)=w_{0}+(1-a) w_{a}$. It is important to note that all the parametrizations are consistent with a cosmological constant.

In Figs. 2 and 3 we show the $2 \sigma$ bands of best-fit models to the data, under different parametrizations of the distance or dark energy EOS. In addition to this outer envelope, we are also interested in the distribution of the individual $w(z)$ models within the $2 \sigma$ bands. We thus study the behavior of the models in the $w-w^{\prime}$ plane, which has been suggested as a natural venue in which to distinguish models [19]. We Monte Carlo 600 scalar potentials, $V(\phi)$, and evolution histories, $w(z)$, within the $2 \sigma$ regime of the best-fit parameters for each of the four fitting functions. In Fig. 4 we plot $w$ and $w^{\prime}$ at $z=0.1$ and $z=0.5$ for each Monte Carlo model, with the scatter of points being $2 \sigma$ consistent with our underlying SN data set.
PHYSICAL REVIEW D 75, 103503 (2007)

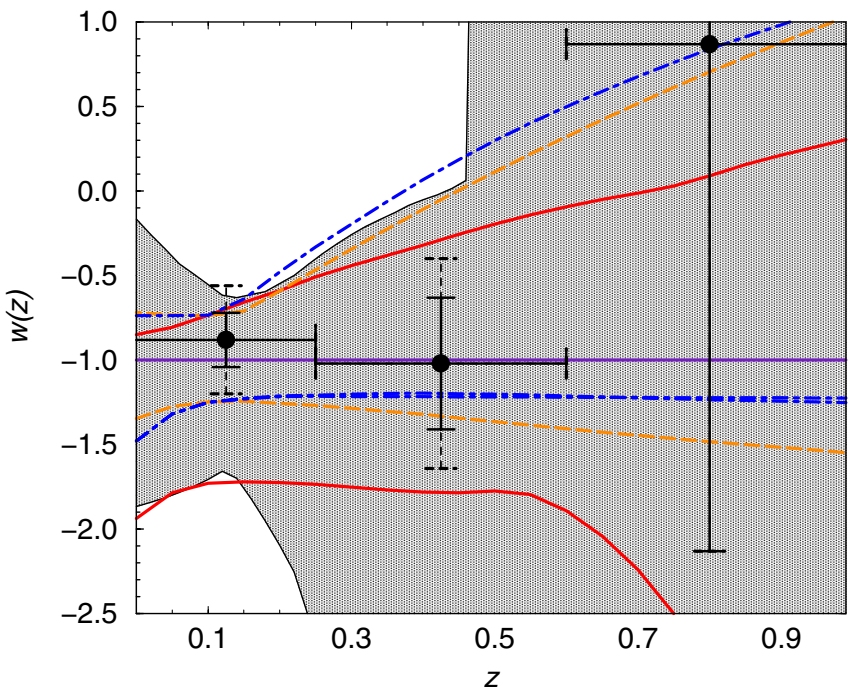

FIG. 3 (color online). The dark energy EOS, $w(z)$, as a function of redshift. The curves show the $2 \sigma$ allowed values and correspond to the potentials shown in Fig. 2. Note that if we impose $\partial \phi / d z>0$, then $w>-1$, as single scalar-field models do not lead to $w<-1$. However, as shown, most of the parametrizations allow the region below $w<-1$. When constructing the potentials shown in Fig. 2, we apply the condition that $\partial \phi / d z>0$. Note that the $w(z)$ parametrizations, with two free parameters, are the most restrictive parametrization in the regime $z<0.3$. Over the redshift range probed, the different parametrizations generally agree with each other. The plotted error bars show the $1 \sigma$ and $2 \sigma$ errors of $w_{i}(z)$ when the EOS is subdivided into three bins in redshift, with $w_{i}(z)$ directly measured from data and no restrictions on its values. A Gaussian prior has been taken on $\Omega_{m}$ with a one sigma uncertainty of 0.05 with $w(z)$ parametrizations.

Based on the evolutionary behavior of simple scalarfield models in the $w-w^{\prime}$ plane, it has been suggested that one can separate potentials into thawing and freezing regions, based upon their shapes [19]. These regions are delineated in Fig. 4, for comparison with our individual Monte Carlo models. It is apparent that the different parametrization approaches yield separate, though often overlapping, regions within the $w-w^{\prime}$ plane. In addition, the models are not necessarily well-contained within the thawing or freezing regions, with a freezing model in one parametrization ending up as a thawing model in another, or with models ending up in between thawing or freezing, or well outside of either regime. Using generic numerical models for the potential shape, this behavior has also recently been highlighted in Ref. [22]. By applying additional constraints on allowable potentials (especially at high z), Ref. [19] finds much tighter confinement in the $w-w^{\prime}$ plane.

Any statement regarding the shape of the scalar potential, as determined from data, is thus crucially dependent upon the underlying parametrizations. For example, for the Taylor expansion approach $w^{\prime}$ is largely negative at $z=0.5$, while it is positive at $z=0.1$. Under the Padé 


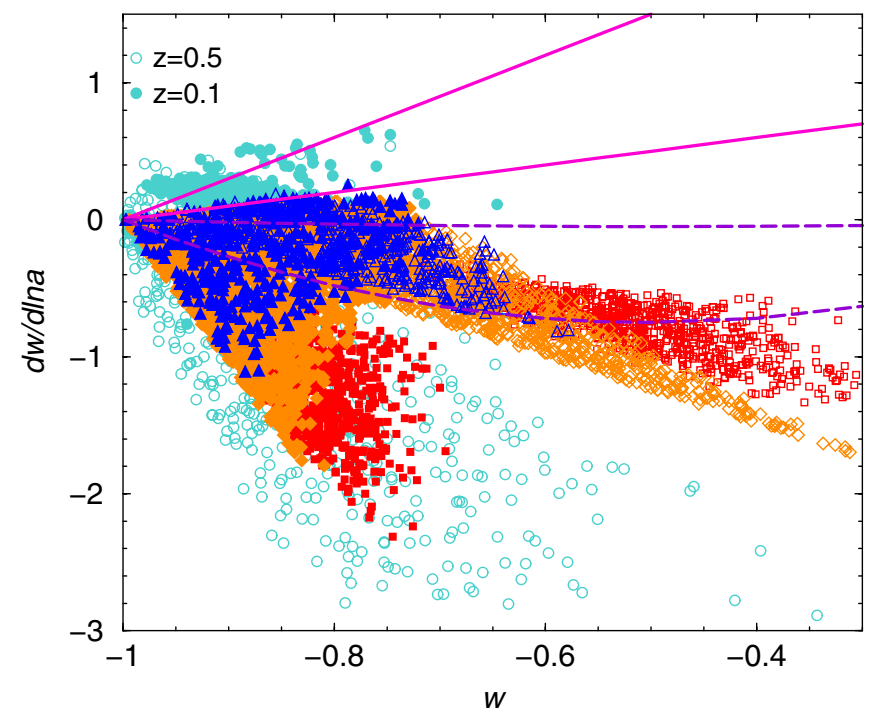

FIG. 4 (color online). $\quad w$ versus $d w / d \ln a$. The data points show the EOS and its time derivative for 600 model potentials uniformly drawn at the $2 \sigma$ confidence level at redshift of 0.1 (filled symbols) and 0.5 (open symbols). The circles, squares, diamonds, and triangle data points show potentials selected under the Taylor expansion, Padé approximation, $w(z)=w_{0}+$ $\alpha \ln (1+z)$ and $w(z)=w_{0}+w_{a}(1-a)$ model fits to the data, respectively. For comparison, we also plot the thawing (dashed) and freezing (solid) potential regions, following Ref. [19]. There are considerable differences in $w$ and $d w / d \ln a$ values, and the evolution captured by two redshifts, between the four approaches. These values do not satisfy the expectations under simple model criteria for the dark energy potentials, though all of these potentials, and the $w(z)$ curves, are consistent with the data at the $2 \sigma$ confidence level.

approximation, $w^{\prime}$ is negative at both $z=0.1$ and $z=0.5$, with $w$ tightly clustered $(-0.9 \lesssim w \lesssim-0.8)$ at $z=0.1$ and relatively unconstrained at $z=0.5$. Although the reconstructed potentials show significant overlap (see Fig. 2), the distributions in the $w-w^{\prime}$ plane are less consistent among different parametrizations. Thus, while there is motivation from theoretical arguments for using the $w$ $w^{\prime}$ plane for potential recognition, there is no obvious, parametrization independent way to convert distance data to constraints in this plane.

The differences seen in Fig. 4 are attributable to the different parametric forms used to approximate the distance or the dark energy EOS. To paraphrase our results: you get out what you put in. Furthermore, the fitting forms to both the distance and the EOS are motivated by their ability to fit data, and possess no clear physical motivation. Figure 4 thus emphasizes the need for an approach which makes minimal assumptions about the underlying potential, thereby maximizing the measurement of a completely unknown scalar field. The less we assume about the potential, the more powerful the ensuing measurement of its shape. Such an approach is presented in the following section.

\section{MODEL-FREE ESTIMATES}

Thus far we have discussed results based on assumed parametrizations for either distance or dark energy EOS. These parametrizations lead to conclusions that are subject to the assumed parametrizations. It is desirable to make model-independent estimates of dark energy. In the case of the EOS $w(z)$, one could approach this by binning $w(z)$ in redshift [21]. Applying this to our SNLS data set, we evaluate $w(z)$ over three bins in redshift, $0<z_{1}<0.25$, $0.25<z_{2}<0.6$, and $0.6<z_{3}<1.0$, assuming $w\left(z_{i}\right)$ constant in each bin.

The resulting best-fit to the $\mathrm{SN}$ data is shown by the data points with $1 \sigma$ and $2 \sigma$ error bars in Fig. 3. We find $w\left(z_{1}\right)=$ $-0.88 \pm 0.28$ and $w\left(z_{2}\right)=-1.02_{-1.26}^{+0.94}$ with no useful constraint for $w(z)$ in the $z_{3}$ bin. Although these bins are correlated at the $10 \%$ level, it is possible to decorrelate the binned $w_{i}(z)$ estimates following the approach of Ref. [21]. While only three bins are attempted here, as SN sample sizes increase, one can consider larger numbers of bins, each narrower in redshift. The estimates shown in Fig. 3 are consistent with estimates based on both fitting functions to the EOS, $w(z)=w_{0}+w_{a}(1-a)$ and $w(z)=$ $w_{0}+\alpha \ln (1+z)$. As discussed in Ref. [21], the binned estimates capture the dark energy EOS with minimal prior assumptions on the parametrization. This is expected to maximize the information one can extract from the data, while minimizing the introduction of biases.

As discussed and noted elsewhere $[8,10]$, the scalar-field potential reconstruction is also subject to prior assumptions on the fitting form. To avoid biases and to make statements that are not subject to assumed parametrization, it is useful to directly construct the potential from data. Recent approaches in the literature consider fitting distance data to a potential expanded as a polynomial in the scalar field with $V(\phi)=\sum_{i=0}^{\infty} V_{i} \phi^{i}[7,22,28]$. Since we are forced to truncate the expansion at low order (for example, at cubic order with existing data [7]), the potential is no longer arbitrary, but rather has a very limited range of possible shapes.

Instead of assuming a specific family of shapes for $V(\phi)$, we propose a "model-free" extraction of the potential directly from the data. We make two assumptions about the scalar-field potential: (1) that it is a piecewise continuous function, and (2) that its structure is "uniform" in the $\phi$ range explored by the data. For $(N-1) \Delta \phi<\phi<$ $N \Delta \phi$, we describe the potential as a function of the field with constant gradients, $d V / d \phi$, over binned intervals, $\Delta \phi$ :

$$
\begin{aligned}
V(\phi)= & V_{0}+\sum_{i=1}^{N-1}(d V / d \phi)_{i} \Delta \phi \\
& +(\phi-(N-1) \Delta \phi)(d V / d \phi)_{N} .
\end{aligned}
$$

Assumption (1) above ensures continuity of $V(\phi)$, which is necessary since one evolves the potential through the dynamic equation for the field as $\ddot{\phi}+3 H \dot{\phi}+d V / d \phi=0$, 
and discontinuities would lead to infinite derivatives. This requirement is unnecessary when considering parameterfree estimates of the dark energy EOS, which is allowed discontinuous jumps in redshift. Both the constant-value and the constant-slope approaches to parametrizing the dark energy EOS lead to similar conclusions [21]. Assumption (2) states that our bins in $\phi$ are fixed width: $\Delta \phi$ is a constant, independent of $\phi$. This assumption could be relaxed (e.g., finer bins near $\phi=0$ ), but this would lead to additional parameters, in addition to introducing modeldependent assumptions into the analysis. The expansion of $V(\phi)$ in Eq. (8) appears to make the least offensive assumptions possible, and thereby offers the basis with which to maximally constrain the full range of possible underlying potentials.

We apply the above potential description to SNLS data following the same approach as Ref. [7], with three free parameters: $V_{0},(d V / d \phi)_{1}$ for $0<\phi<0.03$, and $(d V / d \phi)_{2}$ for $\phi>0.03$. The sizes of the bins are chosen by the range of $\phi$ we are able to constrain, which is in turn related to both the redshift range of the $\mathrm{SN}$ data set and the shape of the potential. Note that we take $\phi=0$ to coincide with $z=0$, but this is an arbitrary choice as we are only sensitive to $\Delta \phi$ over the range of $\Delta z$ of interest. Thus, while we cannot establish the $\phi$ that corresponds to a given $z$ exactly, we can establish the $\Delta \phi$ associated with a given width in redshift. Instead of $(d V / d \phi)_{2}$, we convert the gradient to a data point at $\phi=0.06$, although we find only an upper limit, as this gradient is not strongly constrained by existing data. In Fig. 2 we show the estimated potential and error bars at the $1 \sigma$ and $2 \sigma$ level. The potential values allowed by the data are generally consistent with other indirect reconstructions based on fitting forms for the distance or the EOS. While fitting forms lead to largely positive $V(\phi)$ at $\phi>0.05$, our binned approach finds only an upper limit in this range.

While we have described the potential with only three parameters, this can be straightforwardly generalized to additional bins as the statistics and quality of the SN samples improve. In addition, we make a minimal number of assumptions regarding the potential, and thus are not biased for or against any particular shapes for the scalarfield potential. The proposed approach is similar to the case where the EOS is binned and directly measured from the data without specifying a model for the evolution. As SN data samples increase in size, we believe such a modelindependent approach will become a powerful tool in extracting information about underlying scalar-field potentials.

\section{SUMMARY}

We have presented a reconstruction of a single scalarfield potential using recent SN data from the SNLS survey [13]. We have shown that reconstructions based on various approximations to the distance and the EOS lead to differ- ing evolution histories of the dark energy EOS, particularly when the models are examined in the $w-w^{\prime}$ plane. In this plane the same data can lead to large movements in best-fit models, depending on the specific approximations to distance or EOS which are being utilized. Thus the underlying model assumptions lead to biases, compromising our ability to distinguish evolutionary behaviors of the dark energy. At present the models are only weakly constrained by the data, and thus this model-dependence, although apparent, is not a critical failure. As the data improves, however, a model-independent approach will be essential to determining an otherwise completely unknown scalar-field potential.

As an alternative to existing indirect reconstruction schemes, we have thus proposed a technique which establishes the potential directly from the data, with only minimal assumptions about the underlying shape of the potential. We take the potential to be a binned scalar field, piecewise linear and continuous, but otherwise completely arbitrary. Given the simplicity of these assumptions, this potential is unlikely to introduce biases in the determination of a completely unconstrained, underlying potential. We have demonstrated this approach with current SN data, comparing the results to parametrized analysis. The ensuing constraints, although weaker, are expected to be robust and unbiased. It has been found that direct binning approaches to the dark energy EOS hold great promise for establishing model-independent measurements [21]. We propose a similar approach to reconstructing the underlying dark energy scalar-field potential, allowing us to make assumption-free statements about the nature of the completely unknown and mysterious field potentially responsible for the accelerating expansion of the Universe.

\section{ACKNOWLEDGMENTS}

A. C. and D.E. H. are partially supported by the DOE at LANL through IGPP Grant No. Astro-1603-07. D. E. H. acknowledges support from LANL, and is grateful to the Moore Center for Theoretical Cosmology and Physics at Caltech for its hospitality. C. L. is supported by the Gordon Moore Foundation at Caltech. We thank Dragan Huterer for useful discussions related to this work, and Eric Linder and Robert Caldwell for very detailed comments on an earlier version of the paper.

\section{APPENDIX: ANALYSIS OF ESSENCE+HIGH-Z SNE DATA}

Since the submission of this manuscript, new supernovae results have appeared in the literature, primarily from Hubble Space Telescope observations of type Ia $\mathrm{SNe}$ at $z>1$ [29] and from the Essence survey [30]. The light curves from these two independent data sets have been analyzed with a common method to extract distance moduli in Ref. [31] and we use those data as publicly available 

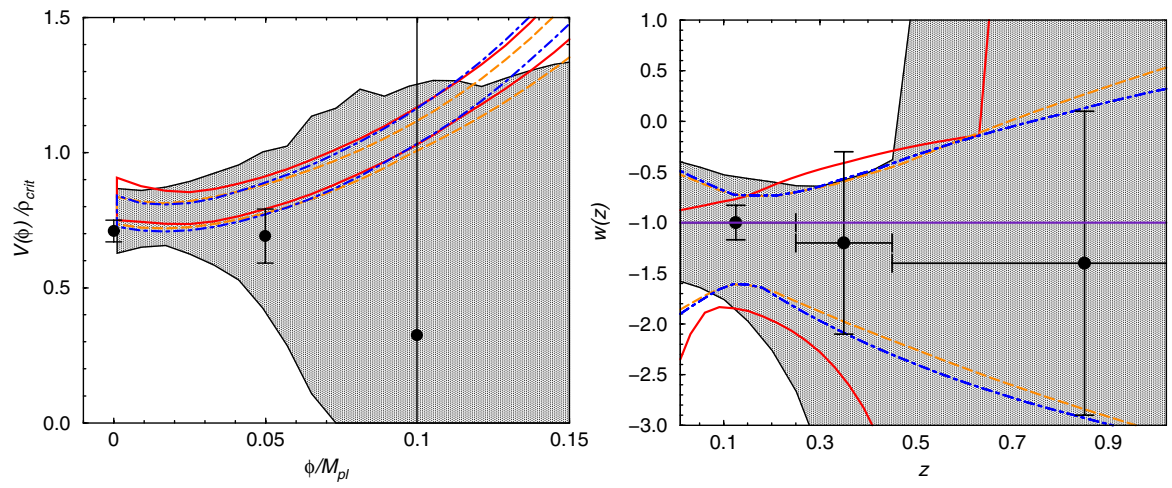

FIG. 5 (color online). The equation of state $w(z)$ (left) and the normalized quintessence potential $\tilde{V}(\phi)$ (right) for Essence + high- $z$ type Ia SNe data. The shaded region is allowed at the $2 \sigma$ confidence level when using the Taylor expansion for $r(z)$. The solid lines mark the same when using a Padé approximation to the distance. The dashed and dot-dashed lines are for the cases where $w(z)$ is parametrized by $w(z)=w_{0}+\alpha \ln (1+z)$ and $w(z)=w_{0}+w_{a}(1-a)$, respectively. The points with error bars show the $1 \sigma$ modelindependent estimates of the potential described in Sec. IV [see Eq. (8)].

[32] to extract equation of state of dark energy and the scalar-field potential.

The methodology is the same as the one outlined in the paper for SNLS data, except that we set $\sigma_{\text {int }}=0.1$ in Eq. (4) following model fitting results of Ref. [31]. We also marginalize over an overall uncertainty in the distance scale, as captured by the Hubble constant. For constant $w$ models, where the equation of state is redshift independent, we reproduce published results by previous analysis of these data. In Fig. 5, we summarize the equation of state (left panel) and the scalar-field potential (right panel) reconstructed by our analysis. Again, we see that the equation of state and the potential is subject to assumptions made with respect to either the polynomial description of distance or the parametric forms of the equation of state. In points with $1 \sigma$ error bars, we also show the nonparametric estimate of either the equation of state or the potential. In the case of ESSENCE + high-redshift supernovae data, the equation of state is such that $w(z)=-1$ at the $1 \sigma$ level over a large range of redshift.
[1] A. Riess et al., Astron. J. 116, 1009 (1998); B. J. Barris et al., Astrophys. J. 602, 571 (2004); R. A. Knop et al., Astrophys. J. 598, 102 (2003); J. L. Tonry et al., Astrophys. J. 594, 1 (2003).

[2] S. Perlmutter et al., Astrophys. J. 517, 565 (1999); A. Riess et al., Astron. J. 116, 1009 (1998).

[3] D. N. Spergel et al. (WMAP Collaboration), Astrophys. J. Suppl. Ser. 148, 175 (2003).

[4] http://www.cfht.hawaii.edu/SNLS/.

[5] http://www.ctio.noao.edu/wproject/.

[6] D. Huterer and M.S. Turner, Phys. Rev. D 60, 081301 (1999).

[7] M. Sahlen, A. R. Liddle, and D. Parkinson, Phys. Rev. D 72, 083511 (2005).

[8] J. Weller and A. Albrecht, Phys. Rev. D 65, 103512 (2002).

[9] I. Maor, R. Brustein, and P. J. Steinhardt, Phys. Rev. Lett. 86, 6 (2001); 87, 049901(E) (2001).

[10] B. F. Gerke and G. Efstathiou, Mon. Not. R. Astron. Soc. 335, 33 (2002).

[11] Z. K. Guo, N. Ohta, and Y.Z. Zhang, Phys. Rev. D 72, 023504 (2005).

[12] V. Sahni and A. Starobinsky, Int. J. Mod. Phys. D 15, 2105 (2006).
[13] P. Astier et al., Astron. Astrophys. 447, 31 (2006).

[14] In the appendix, we also analyze a new set of type Ia $\mathrm{SNe}$ data from the Essence survey complemented with $z>1$ type Ia SNe from the Hubble Space Telescope survey.

[15] T. D. Saini, S. Raychaudhury, V. Sahni, and A. A. Starobinsky, Phys. Rev. Lett. 85, 1162 (2000).

[16] D. Huterer and M. S. Turner, Phys. Rev. D 64, 123527 (2001).

[17] M. Chevallier and D. Polarski Int. J. Mod. Phys. D 10, 213 (2001).

[18] E. V. Linder, Phys. Rev. Lett. 90, 091301 (2003).

[19] R. R. Caldwell and E. V. Linder, Phys. Rev. Lett. 95, 141301 (2005).

[20] Y. Wang and M. Tegmark, Phys. Rev. D 71, 103513 (2005).

[21] D. Huterer and A. Cooray, Phys. Rev. D 71, 023506 (2005).

[22] D. Huterer and H. V. Peiris, astro-ph/0610427.

[23] The distance estimates for two high-redshift $\mathrm{SNe}$ lie more than $3 \sigma$ away from the best-fit relation in the Hubble diagram. As in Ref. [13], we exclude these two data points and only make use of 115 data points to model fit the data.

[24] J. Jonsson, A. Goobar, R. Amanullah, and L. Bergstrom, J. Cosmol. Astropart. Phys. 09 (2004) 007. 
[25] A. G. Kim and R. Miquel, Astropart. Phys. 24, 451 (2006).

[26] A. Cooray, D. Huterer, and D. Holz, Phys. Rev. Lett. 96, 021301 (2006).

[27] L. Hui and P. B. Greene, Phys. Rev. D 73, 123526 (2006); A. Cooray and R. R. Caldwell, Phys. Rev. D 73, 103002 (2006).
[28] M. Sahlen, A. R. Liddle, and D. Parkinson, Phys. Rev. D 75, 023502 (2007).

[29] A. G. Riess et al., astro-ph/0611572.

[30] W. M. Wood-Vasey et al., astro-ph/0701041.

[31] T. M. Davis et al., astro-ph/0701510.

[32] http://www.ctio.noao.edu/wproject/wresults/. 